\title{
EXAMPLES OF COMPLETE MANIFOLDS OF POSITIVE RICCI CURVATURE WITH NILPOTENT ISOMETRY GROUPS
}

\author{
GUOFANG WEI
}

It is well known [4] that the isometry group of a complete riemannian manifold $M$ with strictly positive sectional curvature is always compact. This is no longer true in general when $M$ has Ricci curvature Ric $>0$. The first example was given in [7] for $\operatorname{dim} M=4$. In this note we shall prove

THEOREM. Let $L$ be an $n$-dimensional simply connected nilpotent Lie group. Then for all sufficiently large $p$, the product manifold $M^{p+n}=\mathbf{R}^{p} \times L$ admits complete riemannian metrics with strictly positive Ricci curvature such that the isometry group of $M$ contains $L$.

Using a theorem of Malcev [8], we have as an immediate consequence:

COROLLARY. Every finitely generated torsion-free nilpotent group can be realized as the fundamental group of a complete riemannian manifold with strictly positive Ricci curvature.

On the other hand, every finitely generated subgroup of the fundamental group of any complete manifold with Ric $\geq 0(K \geq 0)$ is nilpotent (abelian) up to finite index $[6,5,4]$.

PROOF OF THE THEOREM. Our construction is inspired by [2]. We first apply an observation in [3, pp. 126-127] to obtain a family of almost flat metrics $g_{r}$ on $L, 0 \leq r<\infty$.

Choose a triangular basis $\left\{X_{1}, \ldots, X_{n}\right\}$ for the Lie algebra $l$ of $L$, i.e., $\left[X, X_{i}\right] \in l_{i-1}$ whenever $X \in l$, and $l_{i-1}$ is spanned by $X_{1}, \ldots, X_{i-1}$. For $X=\sum_{i=1}^{n} a_{i} X_{i}$ set $\|X\|^{2}=\sum_{i=1}^{n} h_{i}^{2}(r) a_{i}^{2}$, where $h_{i}(r)=\left(1+r^{2}\right)^{-\alpha_{i}}$, and $\alpha_{n}=\alpha>0,2 \alpha_{i}-4 \alpha_{i+1}=1,1 \leq i \leq n-1$. The above norm gives rise to a corresponding almost flat left invariant metric $g_{r}$. Then

$$
\left|\operatorname{Ric}_{L}\left(X_{i}\right)\right| \leq c\left(1+r^{2}\right)^{-1},
$$

where $c$ is a constant depending on $n$ and the structure constants.

Now we define a warped product metric $g$ on $M$ by

$$
g=d r^{2}+f^{2}(r) d s^{2}+g_{r}
$$

where $d s^{2}$ is the canonical euclidean metric on the sphere $S^{p-1} \subset \mathbf{R}^{p}, f(r)=$ $r\left(1+r^{2}\right)^{-1 / 4} . g$ is a complete metric on $M$, since $f(0)=0, f^{\prime}(0)=1$, $f^{\prime \prime}(0)=0, f(r)>0$ for $r>0, h_{i}(r)>0$ for $r \geq 0, h_{i}^{\prime}(0)=0$ for $1 \leq i \leq n$.

Received by the editors December 11, 1987.

1980 Mathematics Subject Classification (1985 Revision). Primary 53C20; Secondary $57 \mathrm{~S} 20$. 
It is clear that the isometry group of $g$ contains $L$.

Let $H=\partial / \partial r$ and $U=f(r)^{-1} v$ for a unit tangent vector $v$ of $S^{p-1}$. Straightforward calculation yields:

$$
\begin{array}{ll}
\operatorname{Ric}(H, U)=0, & \\
\operatorname{Ric}\left(X_{i}, H\right)=\operatorname{Ric}\left(X_{i}, U\right)=0, & (1 \leq i \leq n), \\
\operatorname{Ric}\left(X_{i}, X_{j}\right)=0, & (i \neq j, 1 \leq i, j \leq n) .
\end{array}
$$

$\operatorname{Ric}\left(X_{i}, X_{i}\right)=-\frac{g_{i}^{\prime \prime}}{g_{i}}-(p-1) \frac{f^{\prime} g_{i}^{\prime}}{f g_{i}}+\operatorname{Ric}_{L}\left(X_{i}\right)-\sum_{i \neq j} \frac{g_{i}^{\prime} g_{j}^{\prime}}{g_{i} g_{j}}$

$$
\begin{aligned}
\geq\left\{-2 \alpha_{i}\left[\left(2 \alpha_{i}+1\right) r^{2}-1\right]\right. & +(p-1) \alpha_{i}\left(2+r^{2}\right) \\
\left.-c\left(1+r^{2}\right)-\sum_{i \neq j} 4 \alpha_{i} \alpha_{j} r^{2}\right\} /\left(1+r^{2}\right)^{2} & (1 \leq i \leq n) .
\end{aligned}
$$

$$
\begin{aligned}
\operatorname{Ric}(H, H) & =-\sum_{i=1}^{n} \frac{g_{i}^{\prime \prime}}{g_{i}}-(p-1) \frac{f^{\prime \prime}}{f} \\
& =\left\{-\sum_{i=1}^{n} 2 \alpha_{i}\left[\left(2 \alpha_{i}+1\right) r^{2}-1\right]+(p-1) \frac{r^{2}+6}{4}\right\} /\left(1+r^{2}\right)^{2} . \\
\operatorname{Ric}(U, U) & =-\frac{f^{\prime \prime}}{f}+\frac{p-2}{f^{2}}-(p-2)\left(\frac{f^{\prime}}{f}\right)^{2}-\sum_{i=1}^{n} \frac{f^{\prime} g_{i}^{\prime}}{f g_{i}}
\end{aligned}
$$

Since $1-\left(f^{\prime}\right)^{2} \geq 0, f^{\prime \prime} \leq 0$, we have $\operatorname{Ric}(U, U)>0$ in (4). Positivity of the Ricci curvature in the equations (2) and (3) follows for $p$ sufficiently large. Observe that every term of the right-hand side decays at a rate of order at least $r^{-2}$. This completes the proof of the theorem.

REMARK. The smallest $p$ that yields positive Ricci curvature on $M^{p+n}=$ $\mathbf{R}^{p} \times L$ by means of our construction is quite large in general. For example, in the case of the three-dimensional Heisenberg group $L=H^{3}$, we have to choose $p>673$. (With a slightly refined choice of functions, $p>26$ will already work.) We don't know whether or not $p$ can be chosen much smaller. However, it follows from [1] that necessarily $p \geq 4$ when $L=H^{3}$.

ACKNOWLEDGMENT. I would like to thank Detlef Gromoll and Michael Anderson for very helpful discussions.

\section{REFERENCES}

1. M. Anderson, On the topology of complete manifolds of nonnegative Ricci curvature (to appear).

2. L. Bérard Bergery, Quelques exemples de variétés riemanniennes complètes non compactes à courbure de Ricci positive, C. R. Acad. Sci. Paris Sér. A-B 302 (1986), 159-161.

3. P. Buser and H. Karcher, Gromov's almost flat manifolds, Astérisque 81 (1981), 1-148.

4. J. Cheeger and D. Gromoll, On the structure of complete manifolds of nonnegative curvature, Ann. of Math. (2) 96 (1972), 413-443. 
5. M. Gromov, Groups of polynomial growth and expanding maps, Publ. Math. Inst. Hautés Études Sci. 53 (1981), 53-73.

6. J. Milnor, A note on curvature and fundamental group, J. Differential Geom. 2 (1968), 1-7.

7. P. Nabonnand, Sur les variétés riemanniennes complètes à courbure de Ricci positive, C. R. Acad. Sci. Paris Sér. A-B 291 (1980), 591-593.

8. M. S. Raghunathan, Discrete subgroups of Lie groups, Ergeb. Math. Grenzgeb. Band 68, Springer-Verlag, Berlin and New York, 1972.

DePARTMENT OF MATHEMATICS, STATE UNIVERSity OF NEW YORK AT STONY BROOK, STONY BROOK, NEW YORK, 11794 
